\title{
Ectopeptidases, enzymes plurifonctionnelles
}

Les ectopeptidases sont des enzymes protéolytiques transmembranaires présentes dans une grande variété de types cellulaires (nerveux, endocrine, gastro-intestinal, hématopoiétique...) où elles assurent des fonctions variées. Elles dégradent, inactivent ou activent de nombreux facteurs biologiques (peptides intestinaux, hormones, facteurs chimiotactiques, protéines de la matrice extracellulaire...). Elles peuvent jouer un rôle dans l'adhérence cellulaire et peuvent transmettre des signaux d'activation. Enfin, des anomalies de leur expression sont observées dans certaines tumeurs. Ainsi, les ectopeptidases pourraient intervenir dans plusieurs phénomènes : la prolifération, la différenciation, la cytotoxicité, l'adhérence et la migration cellulaire, ce qui explique l'intérêt croissant que suscite l'étude de leur régulation.

\section{Brigitte Bauvois}

\section{ADRESSE}

B. Bauvois : chargée de recherche à l'Inserm. Inserm U. 196, interférons et cytokines, Institut Curie, section de biologie, 26, rue d'Ulm, 75231 Paris Cedex 05, France.

$\mathrm{m} / \mathrm{s} n^{\circ} 5$, vol. 8, mai 92 u mois de mai 1991 s'est tenue au Cnrs PaulLangevin à Aussois (France) la première conférence Jacques Monod résumant les connaissances actuelles sur les ectopeptidases. Les ectopeptidases sont des enzymes protéolytiques présentes à la surface d'une grande variété de cellules de mammiferes, dont le rôle majeur est de dégrader des structures peptidiques et/ou protéiques du milieu extracellulaire. Ce sont des protéines intrinsèques de la membrane cellulaire qui possèdent un seul domaine extracellulaire composé d'une région globulaire glycosylée contenant le site actif de l'enzyme et d'une deuxième région proche de la membrane associée à un site potentiel de clivage.
Selon leur mode d'ancrage à la membrane, les ectopeptidases sont des protéines intégrales de type I (domaine $\mathrm{NH}_{2}$-terminal extracellulaire) ou de type II (domaine $\mathrm{NH}_{2}$-terminal intracytoplasmique).

Au cours des années, un nombre croissant d'ectopeptidases ont été identifiées et l'ensemble de ces enzymes est sous-divisé en deux classes : les endopeptidases, capables de couper toute liaison peptidique située à l'intérieur d'une chaîne polypeptidique et les exopeptidases, qui libèrent un acide aminé ou un dipeptide à partir du groupement $\mathrm{NH}_{2}$ ou $\mathrm{COOH}$ de l'acide aminé terminal [1-11]. Les caractéristiques des principales ectopeptidases (sites de production et cibles physiologiques) sont résumées dans le Tableau I. Sou- 


\section{RÉFÉRENCES}

1. Kenny AJ, Turner AJ. Mammalian Ectoenzymes. Amsterdam : Elsevier Sciences Publishers, 1987.

2. Checler F. Peptidases and neuropeptideinactivating mechanisms in the circulation and in the gastrointestinal tract. In : Danie $\mathrm{EE}$, ed. Neuropeptide Function in the Gastroin testinal Tract. Boca Raton, Boston, Ann Arbor: CRC Press Inc, 1991; 10 273-306.

3. Bauvois B, Gavrilovic J, Wietzerbin J Localizations and potential roles of leukocyte proteases. In : Imhof BA, Berrih-Aknin S Ezine S, eds. Lymphatic Tissues and In Vivo Immune Responses. New York, Basel, Hong Kong : M Dekker Inc, 1991; 43 : 259-65.

4. Naquet P, Pierres M. Cell surface enzymes and lymphocyte functions. Curr Op Immunol 1991 ; 2 : 326-29.

5. Beynon RJ, Bonds JS. Proteolytic Enzy mes : a Practical Approach. Oxford : IRL Press at Oxford Press, 1989.

6. Vijayaraghavan J, Scicli EG, Carretero O, Slaughter C, Moomaw C, Hersh LB "Endothelinase " activity of neutral endopeptidase 24.11. FASEB J 1990 ; 4 : A2301.

7. Pierart ME, Najdovski T, Appelboom TE, Deschodt-Lanckman MM. Effect of human endopeptidase 24.11 (" enkephalinase ") on IL-1-induced thymocyte proliferation activity. I Immunol $1988 ; 140$ $3808-11$.

8. Kounnas MZ, Wolz RL, Gorbea CM Bond JS. Meprin A and B. cell surface endopeptidases of the mouse kidney. $J$ Bio Chem 1991; 266 : 17350-7.

9. Choudry Y, Kenny AJ. Hydrolysis of transforming growth factor- $\alpha$ by cell surface peptidases in vitro. Biochem J $1992 ; 279$ (sous presse).

10. Kreil G, Haiml L, Suchanek G. Stepwise cleavage of the Pro part of promelittin by dipeptidylpeptidase IV. Evidence for a new type of precursor-product conversion Eur $J$ Biochem 1980 ; 111 : 49-58.

11. Bauvois B, Sancéau J, Wietzerbin J Human U937 cell surface peptidase activities : characterization and degradative capacity on tumor necrosis factor- $\alpha$. Eur J Immunol 1992 (sous presse).

12. Hong W, Petell JK, Swank D, Sanford J, Hixson DC, Doyle D. Expression of dipeptidyl peptidase IV in rat tissues is mainly regulated at the mRNA levels. Exp Cell Res 1991; 182 : 256-66.

13. Hildebrandt $M$, Reutter W, Gitlin JD. Tissue-specific regulation of dipeptidyl peptidase expression during development. Biochem J 1991; 277 : 331-4. vent, plusieurs nomenclatures ont été proposées pour une même enzyme. Ainsi, l'endopeptidase 24.11 est nommée CALLA (common acute lymphocytic leukemia antigen) sur la base de sa forte expression sur les cellules leucémiques lymphoïdes alors que, dans le système immunitaire, elle est identifiée à l'antigène CD10 (cluster of differenciation),

La régulation quantitative des ectopeptidases est encore mal connue mais, par analogie avec celle des métalloprotéases sécrétées, ces enzymes doivent être également finement contrôlées. Le clonage des gènes correspondants devrait rapidement permettre une étude détaillée de leur modulation, d'une part, au cours du développement et, d'autre part, lors de phénomènes biologiques tels que l'organisation tissulaire, l'hémostase, la réponse immune et l'inflammation. Déjà, deux études récentes réalisées chez le rat sur l'ectopeptidase dipeptidyl peptidase IV (DPP IV) ont montré que l'expression de cette enzyme au cours du développement est contrôlée à la fois au niveau transcriptionnel [12] et posttranscriptionnel [13]. Par ailleurs, chez la souris, les thymocytes fataux expriment des taux de DPP IV et de $\mathrm{N}$-aminopeptidases très élevés, taux qui chutent rapidement au-delà du $16^{\mathbf{e}}$ jour de développement $[3,4]$. La régulation des ectopeptidases est également sous le contrôle de divers stimuli physiologiques (lectines, cytokines...) et s'exerce au niveau de l'expression de la protéine ancrée à la membrane et de son activité protéolytique (Tableau II, p. 444).

\section{Rôle protéolytique des ectopeptidases}

Les études de protéolyse in vitro de molécules biologiques radiomarquées ont montré que les ectopeptidases caractérisées et identifiées jusqu'ici sont impliquées dans la dégradation d'une large diversité de molécules (Tableau I). Parmi celles-ci, citons des peptides intestinaux (la substance $\mathrm{P}$, la gastrine, le peptide vasointestinal (VIP]), des hormones plasmatiques (les angiotensines, l'insuline, le glucagon), des neurohormones (la neurotensine, la somatostatine, l'ocytocine, les endothélines), des morphi- nes endogènes (les enképhalines), d'autres peptides variés comme la diprotine A (inhibiteur microbien de DPP IV), la bradykinine (peptide algogène) et le peptide chimiotactique pour les neutrophiles (FLMP, formyl Met-Leu-Phe), des cytokines et des facteurs de croissance (interleukines IL- $\alpha, \beta$ et IL- 6, TGF $\alpha$ [transforming growth factor-alpha] TNF $\alpha$, [tumor necrosis factor-alpha] et également des protéines de la matrice extracellulaire (collagène, fibronectine, protéoglycannes). Ainsi, la mise en jeu des fonctions de dégradation par les ectopeptidases conduit-elle à l'activation de prohormones, à l'inactivation d'hormones, de facteurs chimiotactiques et de cytokines mais aussi à la destruction partielle de la matrice extacellulaire. D'après le Tableau I, il apparaît, d'une part, qu'une ectoenzyme donnée est capable de reconnaître une séquence de clivage présente dans différents substrats biologiques et, d'autre part, que des ectoenzymes différentes peuvent dégrader un même facteur biologique bien qu'elles ne le clivent pas au même site (figure 1, p. 444).

Il faut souligner qu'un grand nombre de protéines membranaires sont libérées dans les milieux circulants (urine et sang) selon un processus protéolytique [17]. Ces protéines regroupent des facteurs de croissance (TGF $\alpha$, $\mathrm{TNF} \alpha, \mathrm{CSF} 1$ [colony stimulating factor], EGF [epidermal growth factor] et S-CGF [stem cell growth factor]), des récepteurs pour des cytokines et facteurs de croissance (TNF $\alpha$, NGF [nerve growth factor , CSF-1, IL-1, IL-2 et IFN $\gamma$ [interféron-gamma]) mais aussi des ectoenzymes elles-mêmes (cholinestérase, sialyltransférase, endopeptidase neutre 24.11, dipeptidylpeptidase IV, aminopeptidase- $\mathrm{N}$, dicarboxypeptidase A) [17]. Les enzymes protéolytiques impliquées dans ces mécanismes de clivage n'ont pas encore été identifiées, mais il est possible que les ectopeptidases soient responsables du relargage des protéines solubles.

\section{Rôle des ectopeptidases dans l'adhérence cellulaire à la matrice extracellulaire}

En 1985, les travaux de Hanski et al. [18] suggéraient l'implication de l'ectopeptidase dipeptidylpeptidase IV 
Tableau 1

CARACTÉRISTIQUES DE QUELQUES-UNES DES ECTOPEPTIDASES IDENTIFIÉES

\begin{tabular}{|c|c|c|c|}
\hline Enzyme & Localisation & Substrats & Références \\
\hline $\begin{array}{l}\text { Endopeptidases } \\
\text { Endopeptidase neutre } 24.11 \\
\text { (enképhalinase) } \\
\text { (CALLA) } \\
\text { (CD10) }\end{array}$ & $\begin{array}{l}\text { distribution large et dans le } \\
\text { tissu hématopoiétique exclu- } \\
\text { sivement sur les B et T } \\
\text { immatures, granulocytes }\end{array}$ & $\begin{array}{l}\text { enképhalines, substance } P \text {, } \\
\text { gastrine, tachynine, angiotensi- } \\
\text { nes I, II somatostatine, endo- } \\
\text { théline, ocytocine IL-1 } \beta \text {, IL-6 } \\
\text { tuftsine, f-Met-Leu-Phe, } \\
\text { bradykinine, neurolynine }\end{array}$ & {$[1-7]$} \\
\hline Endopeptidase 2 (méprine) & $\begin{array}{c}\text { distribution restreinte : rein, } \\
\text { intestin, thyroïde glande } \\
\text { salivaires }\end{array}$ & $\begin{array}{c}\text { somatostatine, chaîne B de } \\
\text { I'insuline, IL- } 1 \alpha, \beta, \text { IL- } 6 \text {, } \\
\text { TGF- } \alpha, \text { neurotensine, } \\
\text { bradykinine }\end{array}$ & {$[2-5,8,9]$} \\
\hline Exopeptidases & & & \\
\hline $\begin{array}{l}\text { Aminopeptidase-N } \\
\text { (CD13) }\end{array}$ & $\begin{array}{c}\text { distribution large mais } \\
\text { absente sur les cellules B } \\
\text { et T }\end{array}$ & $\begin{array}{l}\text { enképhalines, angiotensines I, } \\
\text { II, somatostatine, tuftsine, f- } \\
\text { Met-Leu-Phe, thymopentine }\end{array}$ & {$[1-4]$} \\
\hline Aminopeptidase-P & $\begin{array}{l}\text { rein, intestin, absente du } \\
\text { tissu hématopoiétique }\end{array}$ & $\begin{array}{l}\text { substance } P \text {, diprotine } A \text {, } \\
\text { bradykinine }\end{array}$ & {$[1,2]$} \\
\hline Aminopeptidase-A & $\begin{array}{c}\text { distribution large sauf dans } \\
\text { le tissu hématopoiétique : } \\
\text { présente seulement sur les } \\
\text { pré-B }\end{array}$ & & {$[1-4]$} \\
\hline $\begin{array}{c}\text { Dipeptidyl carboxy- } \\
\text { peptidase } A \\
\text { (ACE) } \\
\text { (kininase II) }\end{array}$ & $\begin{array}{c}\text { cellules endothéliales, épi- } \\
\text { théliales, germinales, } \\
\text { monocyte, macrophage, } \\
\text { pré-B }\end{array}$ & $\begin{array}{c}\text { enképhalines substance } P \text {, } \\
\text { angiotensine } I \text {, bradykinine } \\
\text { neurotensine }\end{array}$ & {$[1-3,5]$} \\
\hline $\begin{array}{l}\text { Dipeptidyl amino- } \\
\text { peptidase IV } \\
\text { (CD26) }\end{array}$ & $\begin{array}{c}\text { distribution large mais appa- } \\
\text { remment absente dans le } \\
\text { cerveau }\end{array}$ & $\begin{array}{c}\text { substance } P \text {, promélittine, } \\
\text { neurotensine, collagène I, } \\
\text { TNF- } \alpha\end{array}$ & $\begin{array}{c}{[1-4]} \\
{[10,11]}\end{array}$ \\
\hline
\end{tabular}

IL : interleukine ; TNF : tumor necrosis factor ; TGF : transforming growth factor.

(DPP IV) dans l'attachement in vitro des hépatocytes de rat à une matrice insolubilisée constituée par de la fibronectine et du collagène dénaturé de type I. Une série d'articles a par la suite confirmé cette hypothèse. En utilisant des techniques d'immunohistochimie et de chromatographie d'affinité, il a été montré que la DPP IV des fibroblastes murins et des hépatocytes de rat interagit directement avec le collagène I [19, 20]. De plus, l'inhibition de l'adhérence des cellules au collagène I par une fraction soluble de l'enzyme purifiée et par des anticorps polyclonaux anti$m / s n^{\circ} 5$, vol. 8, mai 92
DPP IV soulignait le rôle fonctionnel d'une telle ectoenzyme dans l'attachement des cellules à la matrice extracellulaire. Il a été récemment montré que la DPP IV peut exprimer une activité endopeptidasique dirigée contre les résidus proline internes insérés dans la séquence répétée X-ProGly des collagènes [3]. Comme l'attachement de l'ectopeptidase au collagène insolubilisé n'altère pas son activité protéolytique, cela implique qu'une telle interaction ne fait pas intervenir le site catalytique de l'enzyme [20]. Dans un autre travail, Piazza et al [21] ont montré que la
DPP IV purifiée se fixe également à la fibronectine par un site différent du site catalytique. Les sites polypeptidiques de la DPP IV, essentiels à la reconnaissance par les cellules de la fibronectine et du collagène, restent à identifier. Le mécanisme potentiel d'action d'une ectopeptidase telle que la DPP IV dans l'adhérence de la cellule à la matrice extracellulaire comprendrait deux étapes (figure 2, p. 445). Lors d'une étape de reconnaissance (liaison), l'ectoenzyme pourrait lier la protéine acceptrice (fibronectine, collagène) sans exprimer d'activité protéolytique, mainte- 


\section{RÉFÉRENCES}

14. Wu $\mathrm{O}$ Li L, Cooper MD, Pierres $M$, Gorvel JP. Aminopeptidase A activity in the murine B-lymphocyte differentiation antigen BP-1/6C3. Proc Natl Acad Sci USA 1991 $88: 676-80$.

15. Lavie G, Zucker-Franklin D. Cellsurface-associated proteinases in NK cellmediated cytotoxicity. Enhancement of enzyme expression is unique to activation with interferon- $\alpha$. Cell Immunol $1989 ; 124$ : 202-11.

16. Werfel T, Sonntag G, Weber $\mathrm{MH}$ Götze $\mathrm{O}$. Rapid increase in the membrane expression of neutral endopeptidase (CD10), aminopeptidase $\mathrm{N}$ (CD 13), tyrosine phosphatase (CD45), and Fcy-RIII (CD16) upon stimulation of human peripheral leukocytes with human C5a. J Immunol 1991; 147 3909-14.

17. Ehlers MRW, Riordan JF. Membrane proteins with soluble counterparts : a role of proteolysis in the release of transmembrane proteins. Biochemistry 1991; 30 : 10066-74

18. Hanski C, Huhle T, Reutter W. Involvement of plasma membrane dipeptidyl peptidase IV in fibronectin-mediated adhesion of cells on collagen. Biol Chem Hoppe-Seyler $1985 ; 366$ : 1169-76.

19. Bauvois B. A collagen-binding glycoprotein on the surface of mouse fibroblasts is identified as dipeptidyl peptidase IV. Biochem J 1988 ; 252: 723-31.

20. Hanski C, Huhle T, Gossrau R, Reutter $W$. Direct evidence for the binding of rat liver DPP IV to collagen in vitro. Exp Cell Res 1988 ; 178 : 64-72.

21. Piazza GA, Callanan HM, Mowery J, Hixson DC. Evidence for a role of dipeptidyl peptidase IV in fibronectin-mediated interactions of hepatocytes with extracellular matrix. Biochem J $1989 ; 262: 327-34$

22. Hegen $M$, Niedobitek $G$, Klein $C E$ Dtein $\mathrm{H}$, Fleisher B. The T cell triggerring molecule Tp103 is associated with dipeptidyl aminopeptidase IV activity. I Immunol $1990 ; 144: 2908-14$

23. Vivier I, Marguet $D$, Naquet $P$, et al. Evidence that thymocyte activating molecule is mouse CD26 (dipeptidyl peptidase IV). $J$ Immunol 1991 ; 147 : 447-54.

24. Dang $\mathrm{NH}$, Torimoto $\mathrm{Y}$, Schlossman SF, Morimoto C. Human CD4 helper T cell activation : functionnal involvement of two distinct collagen receptors $1 \mathrm{~F} 7$ and VLA integrin family. Exp Cell Res $1990 ; 172$
Tableau II

RÉGULATION DE L'EXPRESSION DES ECTOPEPTIDASES PAR DIVERS STIMULI

\begin{tabular}{|c|c|c|c|c|}
\hline \multirow[b]{2}{*}{ Stimulus } & \multicolumn{4}{|c|}{ Amplification de } \\
\hline & $\begin{array}{l}\text { Cellule } \\
\text { cible }\end{array}$ & l'ectopeptidase & $\begin{array}{l}\text { la réponse } \\
\text { cellulaire }\end{array}$ & Référence \\
\hline Con-A, PHA & thymocyte, $T$ & $\begin{array}{c}\text { DPP IV } \\
\text { N-aminopeptidases }\end{array}$ & activation & [3] \\
\hline LPS & B & arginine peptidase & activation & [3] \\
\hline IL-7 & pré-B & AP-A & prolifération & [14] \\
\hline IFN- $\alpha$, IL-2 & NK & $\begin{array}{l}\text { peptidase de type } \\
\text { chymotrypsine }\end{array}$ & cytolyse & [15] \\
\hline $\mathrm{C}_{5} \mathrm{a}$ & granulocyte & $\begin{array}{c}\text { DPP IV } \\
\text { NEP } \\
\text { AP-N }\end{array}$ & activation & [16] \\
\hline
\end{tabular}

AP-A : aminopeptidase $A$; AP-N : aminopeptidase $N$; $C_{5}$ a : facteur anaphylatoxique $C_{5}$; Con-A : concanavaline $A$; DPP IV : dipeptidyl aminopeptidase IV ; LPS : lipopolysaccharide; NEP: endopeptidase neutre 24.11 ; PHA : phytohémagglutinine.

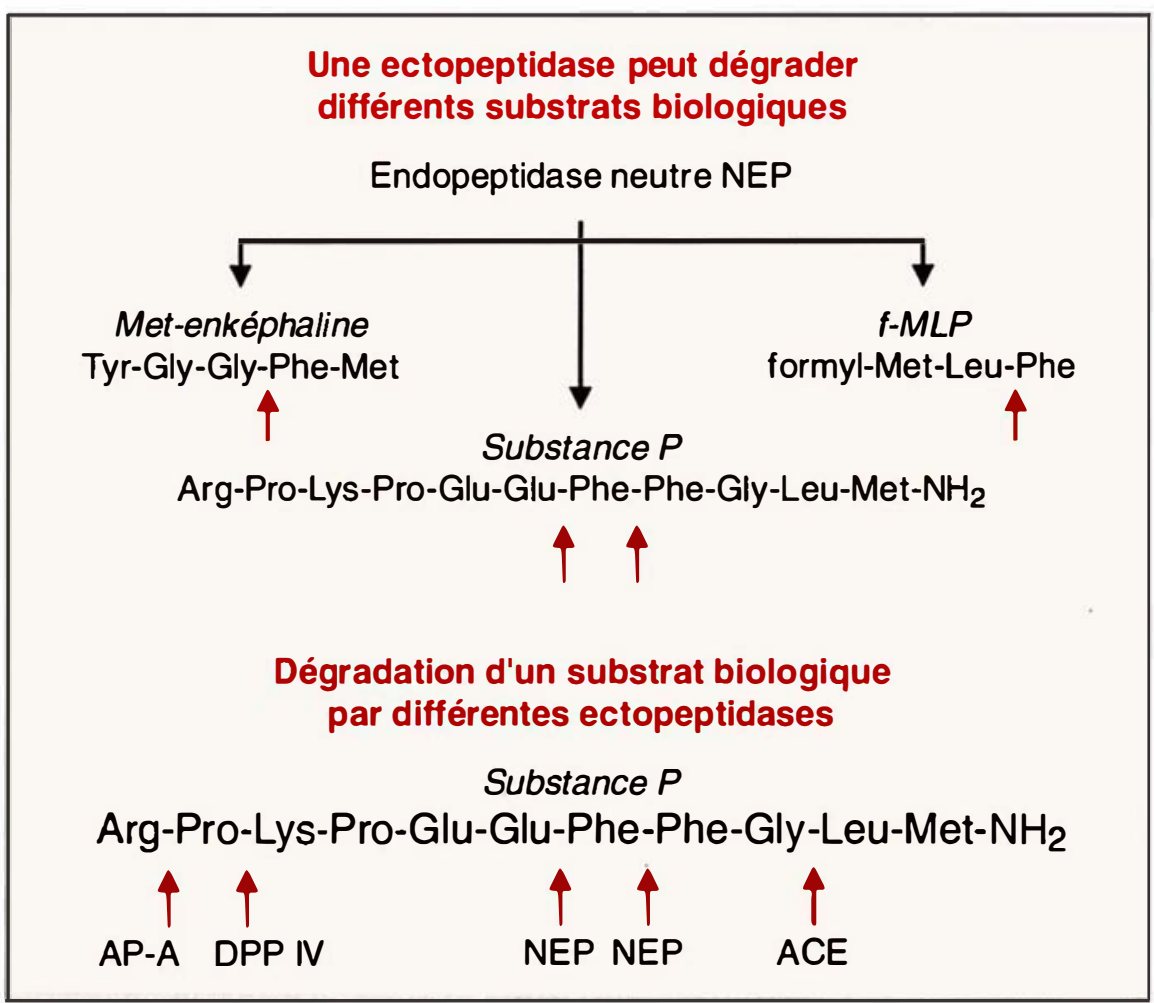

Figure 1. Tableau représentant la polyspécificité des ectopeptidases. $A P$ $A$ : aminopeptidase $A$; DPP $N$ : dipeptidyl aminopeptidase $N$; NEP : endo peptidase neutre 24.11. ACE : dipeptidyl carboxy-peptidase $A$. La flèche noire indique le site de coupure de l'ectoenzyme. 
nant ainsi l'adhérence cellulaire stable. $\mathrm{Si}$, au contraire, l'adhérence est transitoire et réversible, dans une seconde étape, l'ectoenzyme pourrait cliver ou non la protéine matricielle, libérant ainsi la cellule de son support. Le contrôle du fonctionnement de la DPP IV pourrait ainsi autoriser alternativement l'attachement et le détachement cellulaires, mais aussi la migration intervenant au cours de tout phénomène biologique invasif (développement, métastase de la cellule cancéreuse).

\section{Rôle des ectopeptidases dans la signalisation intracellulaire}

D'après ce qui précède, il devient clair que les ectopeptidases peuvent être considérées comme des "récepteurs" spécifiques de divers facteurs biologiques présents dans l'environnement extracellulaire. Ce n'est que très récemment que l'on a découvert que les ectopeptidases pouvaient exercer une action physiologique propre en induisant des événements intracellulaires qui vont conduire à l'activation de la cellule. En 1990, Hegen et al. montraient que des anticorps monoclonaux dirigés contre l'ectopeptidase DPP IV avaient la capacité d'induire une augmentation des capacités proliférative et cytotoxique de cellules humaines $\mathrm{T}$ préactivées [22]. Ces données ont été ensuite confirmées et complétées par des travaux réalisés chez l'humain et chez les rongeurs, sur les cellules de la lignée $\mathrm{T}$ (lymphoblastes thymiques et cellules CD4 + immunocompétentes) [23, 24]. La séquence de l'ectoenzyme réceptrice impliquée dans la transmission des signaux d'activation n'est pas connue. On peut en effet se demander si les sites catalytique et adhésif peuvent être à l'origine de la production du message. Selon les travaux de Shon et al. [25], l'inhibition de l'activité protéolytique de DPP IV par son inhibiteur spécifique (H-Lys$\mathrm{Z}\left(\mathrm{NO}_{2}\right)$-thiazolidide) bloque l'activation cellulaire avec, comme conséquence, une diminution de la production des cytokines IL-2 et IFN- $\gamma$. Cependant, les résultats très récents de Hegen et al. sont en faveur de l'absence de participation du site protéolytique de l'ectoenzyme à l'activa$m / s n^{\circ} 5$, vol. 8, mai 92

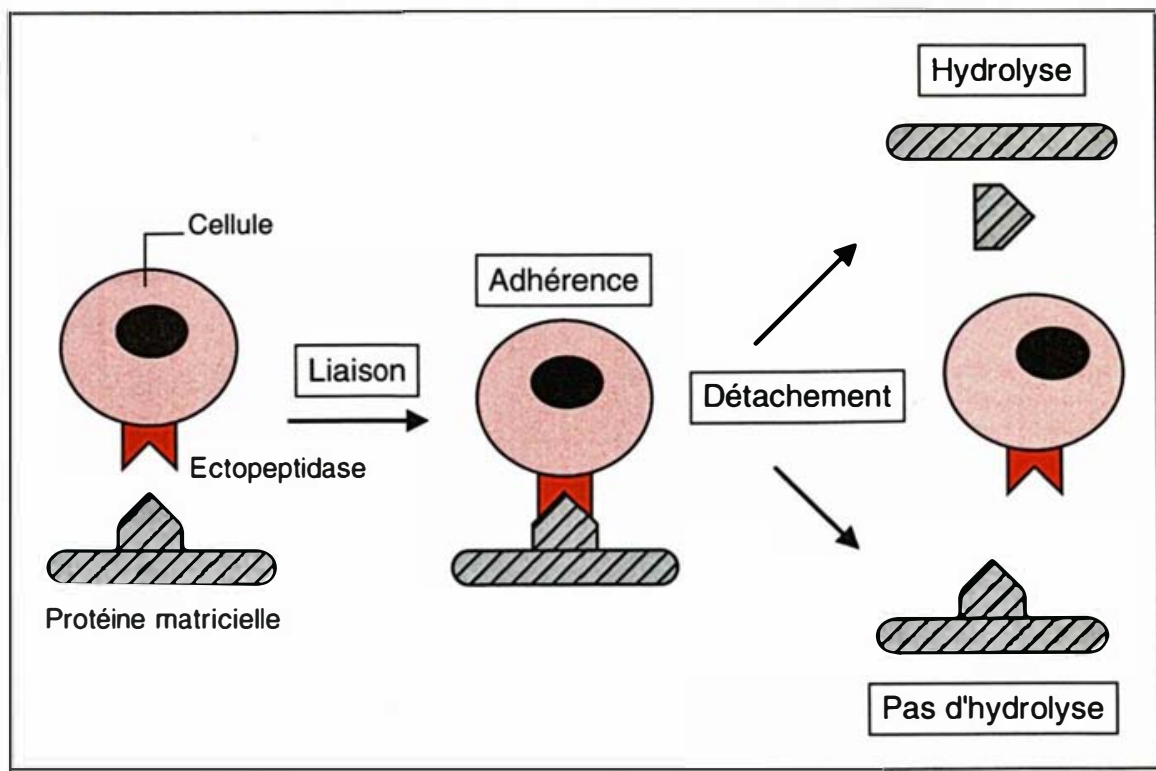

Figure 2. Modèle proposé pour illustrer le rôle des ectopeptidases dans I'adhérence d'une cellule à la matrice extracellulaire.

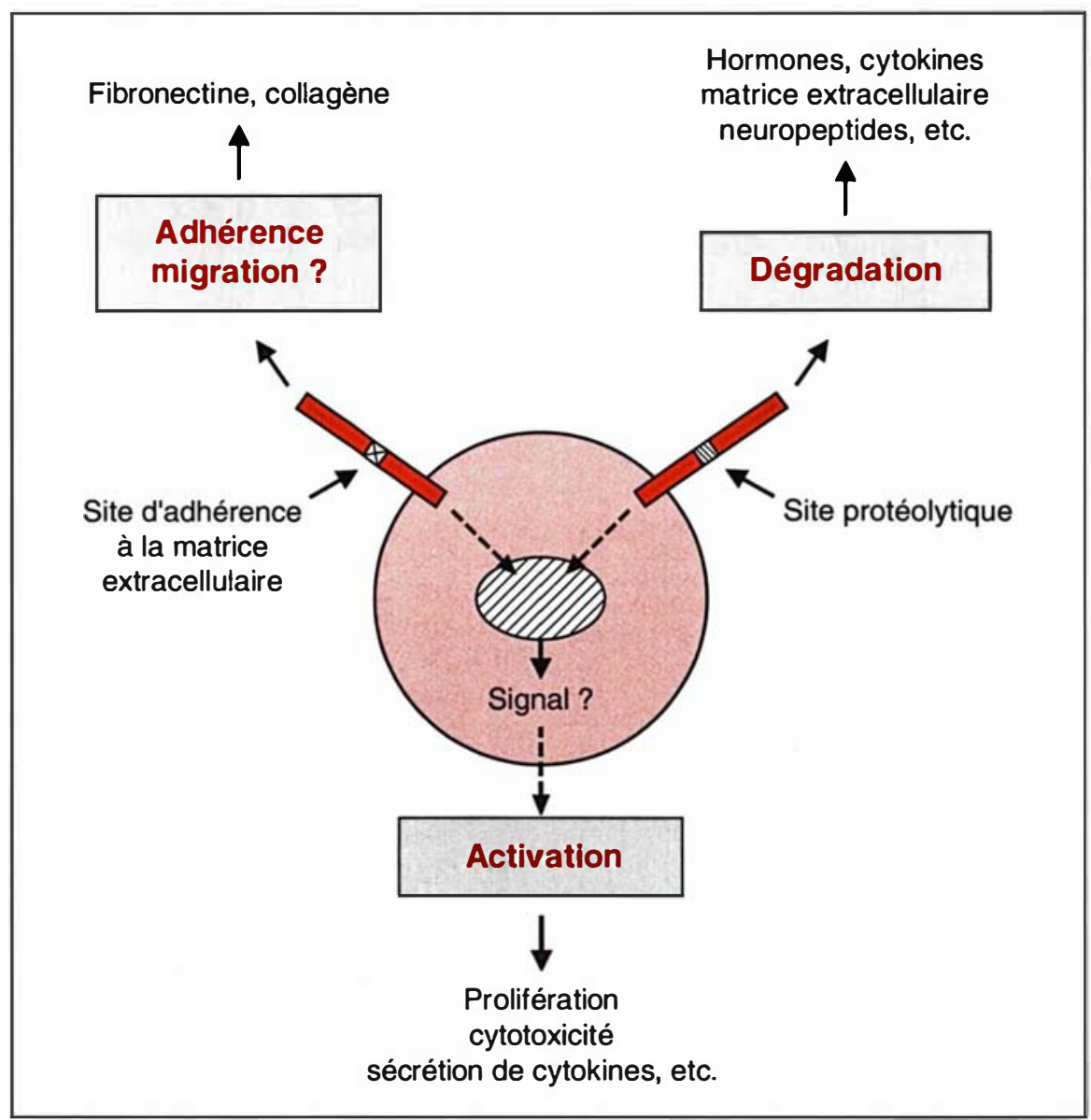

Figure 3. Modèle proposé pour illustrer les différents rôles joués par les ectopeptidases. Les étapes représentées par des lignes noires brisées sont encore mal connues. 


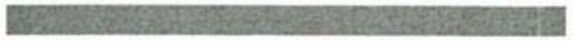

RÉFÉRENCES

25. Schön E, Demuth HU, Eichman E, et al. Dipeptidyl peptidase IV in human $\mathrm{T}$ lymphocytes : impaired induction of interleukin 2 and gamma interferon due to specific inhibition of dipeptidyl peptidase IV. Scand J Immunol 1989 ; 29 : 127-32.

26. Dang $\mathrm{NH}$, Torimoto $\mathrm{Y}$, Shimamura $\mathrm{K}$, et al. 1F7 (CD26): a marker of thymic maturation involved in the differential regulation of the CD3 and CD2 pathways of human thymocyte activation. $J$ Immunol $1991 ; 147$ : 2825-32.

27. Torimoto $\mathrm{Y}$, Dang $\mathrm{NH}$, Vivier $\mathrm{E}$ Tanaka T, Schlossman SF, Morimoto C. Coassociation of CD26 (dipeptidyl peptidase IV) with CD45 on the surface of human T lymphocytes. J Immunol $1991 ; 147$ : 2514-7.

28. Foon KA, Todd RF. Immunologic classification of leukemia and lymphoma. Blood $1986 ; 68: 1-31$.

29. Look AT, Ashumm RA, Shapiro LH Peiper SC. Human myeloid plasma membrane glycoprotein CD13 (gp 150) is identical to aminopeptidase N. J Clin Invest $1989 ; 83$ : 1299-307.

30. Kojima J, Ueno Y, Kasugai H, Okuda $\mathrm{S}$, Akedo H. Glycoproline dipeptidyl aminopeptidase and $\gamma$-glutamyl transpeptidase in human hepatic cancer and embryonal tissues. Clin Chim Acta 1987 ; 167 : 285-91.

31. Scott CS, Stark AN, Minowada J, Drexler HG. Quantitative and qualitative studies of leukaemic cell dipeptidyl peptidases II and IV. Leukemia Res 1988 ; 12 : 129-34.

32. Harland C, Shah T, Webster SADB, Peters TJ. Dipeptidyl peptidase IV subcellular localization, activity and kinetics in lymphocytes from control subjects, immunodeficient patients and cord blood. Clin Exp Immunol $1988 ; 74$ : 201-5.

33. De Pasquale A, Ginaldi L, Limoncell P, Quaglino D. Dipeptidyl amino peptidase IV cytochemistry in circulating lymphocytes from HIV-I-seropositive subjects. Acta Haematol $1989 ; 81$ : 19-21.

34. Aoyama A, Chen WT. A $170 \mathrm{kDa}$ membrane-bound protease is associated with the expression of invasiveness by human malignant melanoma cells. Proc Natl Acad Sci USA 1990 ; 87 : 8296-300.

35. Kido H, Fukutomi A, Katunuma N. A novel membrane-bound serine esterase in human $\mathrm{T} 4$ +lymphocytes immunologically reactive with antibody inhibiting syncytia induced by HIV-1. Purification and characterization. $J$ Biol Chem 1990; 265 :

\begin{tabular}{|c|c|c|c|}
\hline \multirow[b]{3}{*}{ Ectoenzyme } & \multicolumn{3}{|c|}{$\begin{array}{c}\text { Tableau III } \\
\text { OPEPTIDASES ET CELLULES TUMORALES }\end{array}$} \\
\hline & \multicolumn{2}{|c|}{$\begin{array}{l}\text { Expression de la forme } \\
\text { membranaire }\end{array}$} & \multirow{2}{*}{$\begin{array}{l}\text { Expression } \\
\text { augmentée } \\
\text { dans le sérum }\end{array}$} \\
\hline & augmentée & diminuée & \\
\hline NEP & $\begin{array}{l}\text { pré-B-ALL, T-ALL } \\
\text { mélanomes, } \\
\text { gliomes [28] }\end{array}$ & & \\
\hline AP-N & ALL, AML [29] & & \\
\hline DPP IV & $\begin{array}{l}\text { carcinome hépati- } \\
\text { que humain [30] } \\
\text { leucémies post- } \\
\text { thymiques [31] } \\
\text { AML [31] }\end{array}$ & $\begin{array}{l}\text { B-CLL [32] leucé- } \\
\text { mies lymphoïdes } \\
\text { chroniques [32], } \\
\text { lymphocytes de } \\
\text { sujets HIV-I séropo- } \\
\text { sitifs [33] }\end{array}$ & $\begin{array}{l}\text { carcinome hépati- } \\
\text { que humain [30] }\end{array}$ \\
\hline $\begin{array}{l}\boldsymbol{\gamma} \text {-glutamyl } \\
\text { peptidase }\end{array}$ & $\begin{array}{l}\text { carcinome hépati- } \\
\text { que humain [30] }\end{array}$ & & $\begin{array}{l}\text { carcinome hépati- } \\
\text { que humain [30] }\end{array}$ \\
\hline
\end{tabular}

AML : leucémie myéloide aiguë ; $A L L$ : leucémie lymphoide aiguë; AP-N : aminopeptidase-N ; CLL : leucérmie lymphoïde chronique: DPP IV: dipeptidyl peptidase IV: HIV : human immunodeficiency virus NEP : endopeptidase neutre 24.11 tion [communication personnelle]. Il a été également démontré que les cellules $\mathrm{T}$ humaines $\mathrm{CD} 4$ + s'activent en présence du collagène $I$ et que cette activation peut être bloquée par l'addition de peptides Gly-Pro-X (motifs peptidiques répétés dans le collagène) ou par des anticorps monoclonaux anti-DPP IV. Comme nous l'avons précédemment évoqué, il semble que le collagène I soit reconnu au moins par deux domaines distincts de l'ectopeptidase DPP IV : le domaine catalytique et le domaine adhésif. Tenant compte de ces données, la participation éventuelle de l'un de ces deux sites à l'activation cellulaire reste à démontrer. Des seconds messagers induits par l'interaction de l'ectopeptidase avec son (ses) substrat(s) biologique(s) sont en cours d'identification. Dans les lymphocytes $\mathrm{T}$ activés, la stimulation par certains anticorps monoclonaux anti-DPP IV provoque une augmentation des concentrations de calcium intracellulaire [26]. On ne sait cependant pas encore quelles voies d'activation sont mises en jeu (mobilisation du calcium intracellu- laire ou flux entrant de calcium extracellulaire). Des travaux très récents viennent de montrer que DPP IV est physiquement associée à une autre ectoprotéine douée d'une activité tyrosine phosphatasique intracellulaire (CD45 dans le système immunitaire) [27]. Bien que le rôle physiologique de cette protéine dans le mécanisme de transmission du signal par DPP IV ne soit pas connu, il est possible que le complexe ectopeptidase-tyrosine phosphatase soit impliqué dans un phénomène de régulation de la déphosphorylation.

En conclusion, l'ensemble de ces premiers résultats sur la compréhension des mécanismes intracellulaires induits par les ectopeptidases suggère fortement que ces enzymes peuvent participer à la régulation de la prolifération et de la cytotoxité cellulaires ainsi qu'à la sécrétion de cytokines selon le schéma de la figure 3 , page 445.

\section{Conclusions et perspectives}

Il est maintenant bien établi que les ectopeptidases sont présentes sur de 
nombreux types cellulaires, qu'elles peuvent être modulées par des cytokines et qu'elles interviennent à différents stades du développement. Toutes les fonctions potentielles des ectopeptidases évoquées ci-dessus laissent entrevoir l'incidence possible de tels mécanismes dans certaines maladies. Peu d'études cliniques ont cependant abordé l'analyse systématique des ectoenzymes. Une expression anormale de certaines ectopeptidases a été observée dans des hépatocarcinomes, dans des lymphomes B et $T$, dans des myélomes, dans des mélanomes et dans des gliomes (Tableau III) [28-33]. Dans d'autres cas, plusieurs ectopeptidases ont été décrites comme étant exclusivement exprimées par des lignées cellulaires établies à partir de cellules tumorales (lignées T Molt-4 et Jurkat, myélome U266 et mélanome LOX) ou par des cellules transfectées par des virus (exemple des fibroblastes de poulet transfectés avec le virus du sarcome de Rous) [3, 34, 35]. Bien que ces ectoenzymes puissent servir de marqueur des cellules tumorales, aucune corrélation n'a été recherchée entre la présence de telles enzymes et le phénotype invasif de ces cellules. Pourtant, un mauvais fonctionnement de ces ectopeptidases pourrait contribuer à l'invasivité et au pouvoir métastasiant des cellules cancéreuses. Il devient alors important de localiser l'expression de telles enzymes in vivo. La production d'inhibiteurs spécifiques, d'anticorps monoclonaux et le clonage des gènes codant pour les ectopeptidases constituent autant d'outils d'investigation pour l'évaluation des implications biologiques et cliniques des anomalies de fonctionnement des ectopeptidases

\section{Remerciements}

L'auteur remercie les Drs Josette Badet et Jean-Pierre Kolb pour la lecture de ce manuscrit.

\section{TIRÉS A PART}

B. Bauvois.

$\mathrm{m} / \mathrm{s} n^{\circ} 5$, vol. 8, mai 92

\section{Summary}

Involvement of ectopeptidases in varied functions

Ectopeptidases are proteolytic enzymes bound to the surface of immature and mature cells. They are found in a wide variety of tissues and cell types, including immune, nervous, gastrointestinal and endocrine. Their expression is controlled by biologically relevant factors, including cytokines. They have a least three possible function : (a) degradation, (b) specific attachment to the extracellular matrix and (c) signal transduction. In addition to their importance in producing active/inactive effectors from hormones, cytokines and extracellular matrix proteins, ectopeptidases may also be involved in the proteolysis of specific cell surface membrane components. Through the three mentioned above functions, they might be capable of inducing cell proliferation and differentiation, cellmediated cytotoxicity, and cell adhesion and migration. Recent evidence suggests that ectopeptidases play important roles in normal developmental processes and in pathological conditions. 